\title{
Adaptação cultural e validade de conteúdo do ISTAP Skin Tear Classification para o português no Brasil
}

\section{Cultural adaptation and content validity of ISTAP Skin Tear Classification for Portuguese in Brazil}

\section{Adaptación cultural y validez de contenido del ISTAP Skin Tear Classification para portugués en Brasil}

\author{
Cinthia Viana Bandeira da Silva ${ }^{1,2}$, Ticiane Carolina Gonçalves Faustino Campanili2, \\ Kimberly LeBlanc ${ }^{3}$, Sharon Baranoski ${ }^{4}$, Vera Lúcia Conceição de Gouveia Santos ${ }^{5}$
}

\section{ORCID IDS}

Silva CVB (D) https://orcid.org/0000-0003-1430-1237 Campanili TCGF (D) https://orcid.org/0000-0003-4134-6035 LeBlanc K (D) https://orcid.org/0000-0001-5003-686X Santos VLCG (D) https://orcid.org/0000-0002-1288-5761

\section{COMO CITAR}

Silva CVB; Campanili TCGF; LeBlanc K; Baranoski S; Santos VLCG. Adaptação cultural e validade de conteúdo do ISTAP Skin Tear Classification para o português no Brasil. ESTIMA, Braz.J. Enterostomal Ther., 16: e2618. doi: 10.30886/estima.v16.590_PT.

\begin{abstract}
RESUMO
Objetivo: Adaptar culturalmente o ISTAP Skin Tear Classification para a língua portuguesa no Brasil e testar a validade de conteúdo da versão adaptada. Métodos: Três fases compuseram a adaptação cultural: tradução, avaliação por comitê de juízes composto de cinco estomaterapeutas (gerando a validação de conteúdo do instrumento) e retrotradução. O projeto foi aprovado por comitê de ética em pesquisa. Resultados: Duas versões em português do instrumento foram obtidas após tradução e analisadas pelo comitê de juízes, ocorrendo discordâncias apenas em termos específicos utilizados na área, seguidas por sugestões para melhor adequação do vocabulário em saúde. Isso gerou valores baixos do índice de validade de conteúdo. No entanto, a validade de conteúdo foi confirmada após discussão das discrepâncias entre as autoras e alguns membros do comitê de juízes, bem como com uma das autoras do instrumento original, Dra. Kimberly LeBlanc, que também a atestou quando aprovou as retrotraduções dessa versão. Conclusão: Considera-se obtida a versão adaptada culturalmente do ISTAP Skin Tear Classification, com sua validade de conteúdo também atestada. Neste momento, os testes para confiabilidade inter e intraobservadores e validade concorrente estão em fase de finalização, após o que se disponibilizará o instrumento adaptado e validado para o Brasil.
\end{abstract}

DESCRITORES: Estudos de validação; Ferimentos e lesões; Pesquisa metodológica em enfermagem; Prática avançada de enfermagem.

\footnotetext{
1 Universidade de São Paulo - Faculdade de Medicina - Instituto do Coração do Hospital das Clínicas - São Paulo/SP - Brasil.

Universidade de São Paulo - Escola de Enfermagem - São Paulo/SP - Brasil.

3Western University - Scholl of Physical Therapy - Advanced Health Care Practice Program - Ontario - Canada.

${ }^{4}$ National Pressure Ulcer Advisory Panel - Washington, DC - EUA.

5Universidade de São Paulo - Escola de Enfermagem - Departamento de Enfermagem Médico-Cirúrgica - São Paulo/SP - Brasil. Autor correspondente: Cinthia Viana Bandeira da Silva | Rua Sebastião Dias, 38 | CEP: 02976-050 - São Paulo/SP - Brasil | E-mail: bandeira.cinthia@gmail.com

Recebido: Mar. 072018 | Aceito: Jun. 282018
} 


\begin{abstract}
Objective: To translate and culturally adapt the International Skin Tear Advisory Panel (ISTAP) Skin Tear Classification into the Portuguese language in Brazil and test the content validity of the adapted version. Methods: The cultural adaption comprised three phases: translation, evaluation by committee of judges composed of five stomatherapists (confirming the instrument content validity) and back-translation. Results: Two Brazilian Portuguese versions of the instrument were obtained after translation and analyzed by the committee, disagreements arose over several health related terms. This generated low values of the content validity index. However, the content validity was confirmed after discussion of discrepancies between the authors and some members of the judges' committee, as well as with one of the authors of the original instrument, Dr. Kimberly LeBlanc, who also testified that validity when approving the back-translations of the adapted version to Brazilian Portuguese. Conclusion: The culturally adapted version of the ISTAP Skin Tear Classification is considered to have been obtained, with its content validity also attested. At that moment, the tests for inter and intraobserver reliability and concurrent validity are in the finalization phase, after which the instrument adapted and validated for Brazil will be made available.
\end{abstract}

DESCRIPTORS: Validation studies; Wounds and injuries; Methodological research in nursing; Advanced nursing practice.

\begin{abstract}
RESUMEN
Objetivo: Adaptar culturalmente el ISTAP Skin Tear Classification para la lengua portuguesa en Brasil y probar la validez de contenido de la versión adaptada. Métodos: Tres etapas constituyeron la adaptación cultural: traducción, evaluación por comité de jueces compuesto por cinco estomaterapeutas (generando la validación de contenido del instrumento) y traducción inversa. El proyecto fue aprobado por comité de ética en investigación. Resultados: Se obtuvieron dos versiones en portugués del instrumento después de ser traducidas y analizadas por el comité de jueces, habiendo discordancias solamente en términos específicos utilizados en el área, seguidas por sugerencias para mejor adecuación del vocabulario de salud. Eso generó valores bajos del índice de validez de contenido. Sin embargo, la validez de contenido fue confirmada después de discusión de las discrepancias entre las autoras y algunos miembros del comité de jueces, así como con una de las autoras del instrumento original, Dra. Kimberly LeBlanc, que también la certificó cuando aprobó las traducciones inversas de esa versión. Conclusión: Se considera obtenida la versión adaptada culturalmente del ISTAP Skin Tear Classification, también con comprobación de su validez de contenido. En este momento, las pruebas para confiabilidad inter e intraobservadores y validez coincidente están en etapa de finalización, después de lo que se hará disponible el instrumento adaptado y validado para Brasil.
\end{abstract}

DESCRIPTORES: Estudios de validación; Heridas y lesiones; Investigación metodológica en enfermería; Práctica avanzada de enfermería.

\title{
INTRODUÇÃO
}

Lesões por fricção (LFs) são feridas traumáticas que ocorrem principalmente nas extremidades e em idosos, causadas por cisalhamento, friç̧ão e/ou força bruta, resultando na separação das camadas da pele. Essa separação pode ser de espessura parcial, quando a derme e a epiderme se separam, ou de espessura total, quando a derme, a epiderme e a estrutura subjacente são separadas entre $\mathrm{si}^{1,2}$.

Já é sabido que alguns fatores alteram o processo de cicatrização e esses apresentam-se, também, como fatores de risco para o aparecimento das LFs, como: extremos de idade, principalmente idosos com mais de 85 anos; sexo feminino e caucasianos; estado nutricional prejudicado; uso de medicamentos, tais como imunossupressores, agentes anti-inflamatórios e anticoagulantes, esse último por causar, frequentemente, o aparecimento de equimoses; tabagismo; perda da sensibilidade; dependência para atividades de vida diária; mobilidade prejudicada; e estado da doença atual ${ }^{1,3}$.
Embora esse tipo de lesão não seja recente, é atual a discussão sobre sua nomenclatura. A necessidade de sistematizar o processo de avaliação das LFs levou à iniciativa pioneira de Payne e Martin ${ }^{4,5}$,em 1990, quando desenvolveram o Payne-Martin Classification System for Skin Tears. Embora sem propriedades de medida testadas, o sistema foi e ainda é bastante utilizado ${ }^{6}$. A partir do reconhecimento da necessidade e importância dessa padronização, somente 15 anos mais tarde, dois grupos internacionais distintos passaram a propor novos sistemas de classificação, ambos baseados na primeira iniciativa de Payne-Martin: STAR Skin Tear Classification System², em 2007, e International Skin Tear Advisory Panel (ISTAP)', em 2011.

No Brasil, publicou-se, recentemente, o processo de adaptação cultural e validação do sistema de classificação STAR Skin Tear Classification System ${ }^{7}$, introduzindo-se a nova nomenclatura - lesão por fricção - em substituição 
à anterior, laceração ou ferida traumática, considerada mais genérica. Desenvolvido por um grupo de enfermeiros australianos especialistas no tratamento de feridas e coordenado pela Prof. ${ }^{a}$ Dr ${ }^{\mathrm{a}}$. Kerylin Carville, o STAR é constituído de guia de tratamento, sistema de classificação e glossário. O guia de tratamento apresenta seis tópicos direcionados para o cuidado com a ferida e a pele ao redor, e o sistema de classificação avalia a presença/ausência do retalho de pele e sua viabilidade em cinco fotografias, cada qual relacionada à sua respectiva descrição categórica. $\mathrm{O}$ glossário, por sua vez, traz definições das LFs e os termos técnicos relacionados a elas ${ }^{2}$.

O ISTAP, originalmente coordenado pela canadense Dra. Kimberly LeBlanc e pela norte americana Dra. Sharon Baranoski, é também responsável pelo desenvolvimento de consenso sobre diagnóstico, tratamento e prevenção dessas lesões agudas traumáticas. A decisão pelo seu desenvolvimento partiu de levantamento realizado em 16 países, com 1.127 profissionais de saúde, dos quais 69,6\% alegaram dificuldades em estabelecer a avaliação e a documentação das $L^{2}{ }^{1}$. O consenso foi elaborado, primeiramente, por um painel composto de 13 especialistas em tratamento de feridas procedentes dos Estados Unidos da América (EUA), Canadá, Reino Unido e Austrália. Após a elaboração desse consenso, os membros contaram com a revisão final por um painel consultivo composto de 68 colaboradores externos internacionais, também especialistas no tratamento de feridas. Ao final, os membros de ambos os painéis finalizaram o consenso constituído de 12 declarações sobre avaliação, predição, prevenção e tratamento das LFs. O consenso incluiu, ainda, um novo sistema de classificação, ISTAP Skin Tear Classification, mais simples, que pudesse ser rapidamente incorporado à prática clínica. Similarmente aos demais sistemas apresentados anteriormente, o ISTAP Skin Tear Classification consta de associação de fotografias, nesse caso, três tipos de lesões caracterizadas como ruptura linear com retalho totalmente presente, perda parcial do retalho e perda total do retalho (Fig. 1$)^{1}$.

A validade interna do ISTAP Skin Tear Classification foi estabelecida por meio de avaliação simultânea de fotografias por especialistas. Assim, um banco original contendo 74 fotografias de alta qualidade, fornecidas pelos demais 12 membros do painel que elaboraram o consenso, foi avaliado pela Dra. LeBlanc após devido consentimento dos autores das fotos. Dentre essas, LeBlanc selecionou 30; após essa seleção, as fotografias foram enviadas aos membros do painel para testar a validade interna do instrumento. Eles foram orientados a agrupar as fotografias em três grupos, de acordo com a similaridade das características, sem referência ao sistema de classificação proposto ${ }^{8}$.

A estabilidade foi testada dois meses depois, repetindo o processo anterior, com concordância satisfatória (Cohen $\mathrm{k}=0,877$ ). Para validade externa, o estudo utilizou amostra de 190 indivíduos (enfermeiros e profissionais de outras áreas da saúde) que passaram pelo mesmo processo de agrupamento das fotografias, novamente sem se referir ao

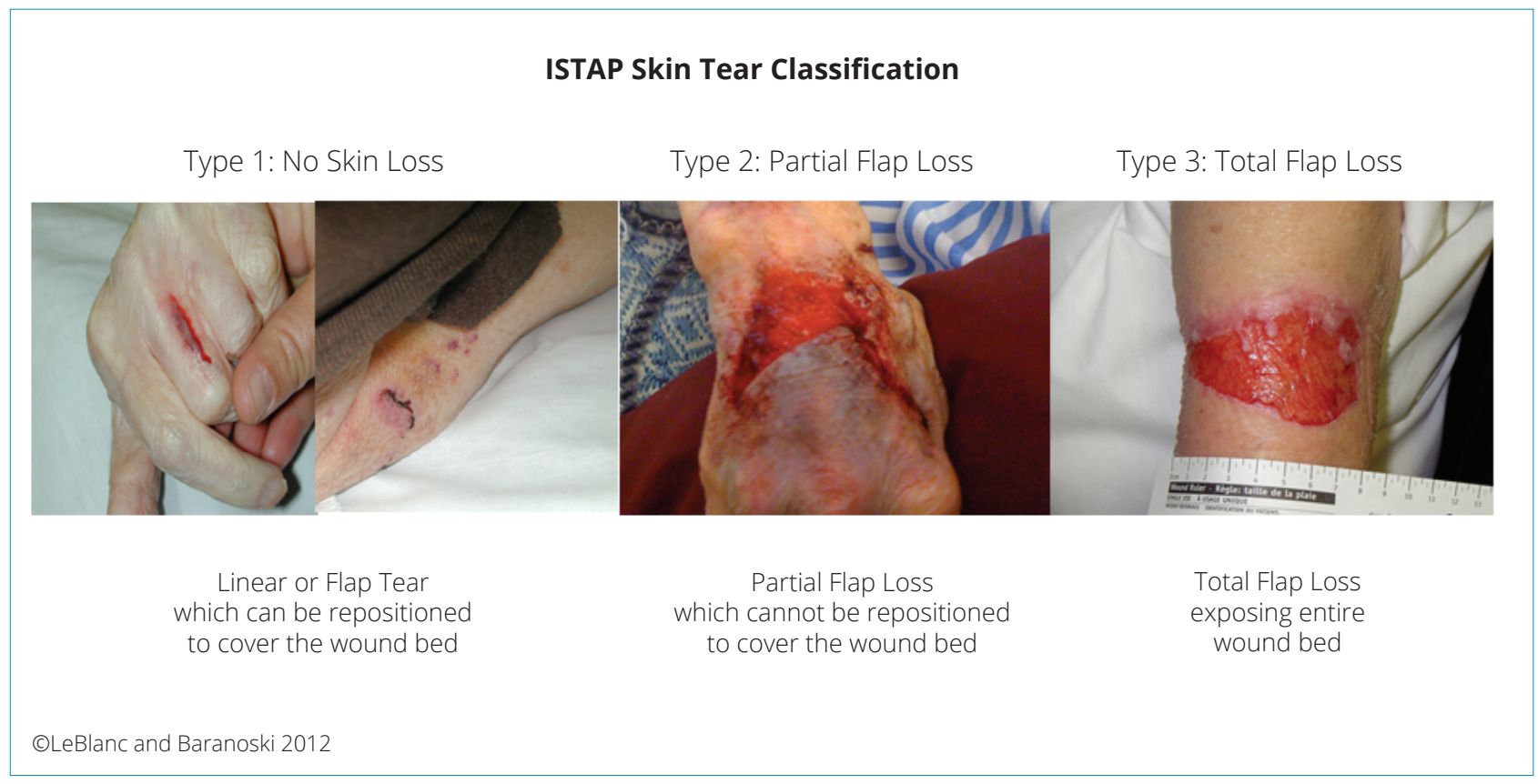

Figura 1. ISTAP Skin Tear Classification ${ }^{8}$ (reprodução autorizada pela autora principal). 
sistema de classificação, ou seja, somente por similaridade de características, obtendo-se nível moderado de concordância $(\text { Fleiss } \mathrm{k}=0,545)^{8}$.

As LFs são lesões pouco estudadas e, consequentemente, subnotificadas, sendo frequentemente confundidas com lesões por pressão. O Brasil é um dos poucos países latinoamericanos com estudos publicados a respeito das LFs e, ainda assim, essas lesões são pouco conhecidas na prática clínica. A adaptação e a validação de um segundo sistema internacional de classificação simples e acessível, como o ISTAP Skin Tear Classification, permitirão uma linguagem sistematizada comum, facilitando a comunicação entre os profissionais de saúde, o planejamento mais adequado da assistência a ser prestada, bem como a comparação dos resultados de nossas publicações com aqueles alcançados por autores internacionais. O objetivo deste estudo é apresentar apenas a etapa de adaptação cultural do ISTAP Skin Tear Classification, além da análise da validade de conteúdo da versão adaptada, como um dos itens do processo de adaptação cultural de instrumentos de medida.

\section{MÉTODOS}

Trata-se de estudo metodológico com abordagem quantitativa sobre a adaptação cultural do ISTAP Skin Tear Classification baseado na proposta de Beaton et al. ${ }^{9}$.

O convite e a autorização para adaptação e validação do ISTAP Skin Tear Classification para o português no Brasil foram feitos pela Dra Kimberly LeBlanc, coordenadora do painel internacional ISTAP, à Prof. ${ }^{a}$ Dra. Vera Lucia C. G. Santos. O Comitê de Ética em Pesquisa da Escola de Enfermagem da Universidade de São Paulo (EEUSP) aprovou o projeto (processo 66513517.7.0000.5392/2017/ CEPEEUSP), assim como o Instituto do Coração (InCor) do Hospital das Clínicas da Faculdade de Medicina da USP, local em que se realizou a coleta de dados para avaliação das propriedades de medida da versão adaptada do instrumento. Os enfermeiros e pacientes participantes foram incluídos na amostra do estudo somente após o consentimento e a garantia de anonimato. Embora o presente estudo se relacione somente à etapa de adaptação cultural do ISTAP Skin Tear Classification para o português no Brasil, o projeto foi aprovado como um todo nas instâncias descritas anteriormente. $\mathrm{O}$ estudo foi desenvolvido como trabalho de conclusão do Programa de Residência em
Enfermagem em Cardiopneumologia de Alta Complexidade da EEUSP-InCor.

O processo de adaptação incluiu três etapas: tradução, análise por comitê de juízes (em que se testou também a validade de conteúdo) e retrotradução.

Primeiramente, realizou-se a tradução do ISTAP Skin Tear Classification por dois tradutores brasileiros, fluentes em inglês: um leigo na área da saúde, sem conhecimento do projeto de pesquisa, e um enfermeiro ciente do objetivo do estudo ${ }^{9}$. As traduções foram denominadas, respectivamente, de T1 e T2. Ambos classificaram cada item do instrumento quanto ao grau de dificuldade no processo de tradução ( 0 a 10).

As duas traduções foram analisadas, em seguida, por um comitê de juízes composto de cinco enfermeiras especialistas em estomaterapia, brasileiras, fluentes em inglês, experientes no tratamento de pacientes com feridas e com conhecimento dos conceitos do instrumento. O comitê de juízes é parte fundamental para alcançar a equivalência intercultural do instrumento traduzido e sua avaliação visa à análise das equivalências semântica (correspondência gramatical e de significação dos vocábulos), idiomática (substituição das expressões coloquiais específicas, raramente traduzíveis, por termos equivalentes), cultural (coerência entre os termos utilizados e a cultura da população à qual se destina a aplicação do instrumento) e conceitual (se os termos traduzidos refletem os mesmos conceitos dos originais) das versões traduzidas e a versão original ${ }^{9}$. Cada juiz procedeu à análise utilizando escala tipo Likert (1 a 4) para cada etapa da tradução (respectivamente: discordo totalmente, discordo, concordo e concordo totalmente). Além dessa análise, os membros do comitê foram solicitados a sugerir alterações para adequação e melhoria da redação dos itens visando ao melhor ajuste das traduções realizadas à área da saúde. A concordância entre as respostas dos juízes foi analisada por meio do índice de validade de conteúdo (IVC) obtido pela proporção de itens que recebeu pontuação 3 ou 4 dos juízes ${ }^{10-13}$.

A validade de conteúdo indica em que medida o instrumento tem uma amostra apropriada de itens para medir o construto específico e cobrir adequadamente seu domínio. É cada vez mais comum o uso de um grupo de especialistas independentes para avaliar a validade de conteúdo de instrumentos novos. O IVC indica em que medida as opiniões dos especialistas são congruentes, medindo a proporção ou porcentagem de juízes com concordância sobre os aspectos e os itens do instrumento. Além de analisar 
os itens, permite analisar o instrumento como um todo. A excelência da validade de conteúdo de uma escala é alcançada com um IVC de 0,9010-13.

A partir das análises dos juízes, uma versão única foi estabelecida pelas pesquisadoras, sendo discutidas as discrepâncias entre elas com alguns dos membros do comitê e com a própria autora do instrumento original, Dra. LeBlanc. Essa versão resultante foi então encaminhada à retrotradução, também realizada por dois tradutores, porém ambos leigos na área da saúde e que tinham o inglês como língua materna. Ambas as versões retrotraduzidas foram encaminhadas à autora original do instrumento para confirmar a equivalência entre as versões original e a adaptada.

Os dados compilados foram agrupados em planilhas Excel e analisado por meio do Programa Statistical Package for Social Sciences (SPSS) versão 22.0.

\section{RESULTADOS}

\section{Tradução}

Duas versões do instrumento em português foram obtidas (T1 e T2). Para o tradutor leigo na área da saúde (T1), tanto o título quanto a definição do primeiro tipo de lesão apresentaram maiores graus de dificuldade para serem traduzidos, classificado como 8, justificado pela falta de conhecimento na área da saúde. Já a tradutora que conhecia o objetivo do estudo (T2) referiu dificuldade apenas ao traduzir o termo tear. As duas versões ainda apresentaram pequenas diferenças quanto às traduções dos termos flap e wound bed: flap foi traduzido como aba e borda e wound bed como leito e base da ferida.

\section{Análise do comitê de juízes}

O IVC médio geral foi de 0,66 , quando calculado de acordo com a média das proporções (Tabela 1 ).

As traduções com maiores valores de $\operatorname{IVC}(0,8$ e 1) são do título do instrumento (Classificação ISTAP para lesões por fricção) e do tipo 1 (sem perda de pele). Todas as demais traduções obtiveram sugestões para adequação dos termos traduzidos.

A palavra flap gerou discordância relevante entre os juízes e os tradutores em todos os itens em que apareceu, reduzindo os valores de IVC. A tradução incorreta desse termo levou os juízes à sugestão do emprego da terminologia "retalho cutâneo" em vez de "aba” ou "borda”, indicadas nas traduções.
Tabela 1. Índice De Validade De Conteúdo (IVC) das versões do instrumento traduzidas (T1 e T2) para o português. São Paulo, SP, Brasil, 2017.

\begin{tabular}{|c|c|c|c|}
\hline \multirow{2}{*}{ ISTAP Classification - item } & \multicolumn{2}{|c|}{ IVC } & \multirow{2}{*}{$\begin{array}{l}\text { IVC médio } \\
\text { total }\end{array}$} \\
\hline & $\mathrm{T} 1 *$ & $\mathrm{~T} 2 *$ & \\
\hline Título do instrumento & 0,2 & 0,8 & \\
\hline Título da LF tipo 1 & 1 & 1 & \\
\hline Definição LF tipo 1 & 0,2 & 0,4 & \\
\hline Título da LF tipo 2 & 0 & 0,6 & 0,66 \\
\hline Definição LF tipo 2 & 0,6 & 0,2 & \\
\hline Título da LF tipo 3 & 0,4 & 0,4 & \\
\hline Definição LF tipo 3 & 0,6 & 0,4 & \\
\hline
\end{tabular}

Outro importante item de discussão foi a definição do tipo 1 de LF (IVC: 0,4), que agrupa dois subtipos de LF (ruptura linear e retalho cutâneo - ambos sem perda de tecido cutâneo ou pele) e que, portanto, leva a duas diferentes formas de "recobertura" da lesão (linear or flap tear that can be repositioned to cover the wound bed). Nesse caso, as pesquisadoras principais optaram por discutir o conteúdo da definição com alguns dos membros do comitê, com o tradutor não leigo, bem como com uma das autoras do projeto ISTAP, Dra. LeBlanc. As discussões resultaram em uma definição que incluiu duas formas de recobertura a partir do subtipo (linear ou com retalho): "reaproximação de bordas" ou "reposicionamento do retalho", respectivamente.

Para obter a versão T3, ao final dessa fase, consideraram-se as sugestões dos juízes e estabeleceu-se que o termo "lesão" seria padronizado em todo o instrumento, em vez da palavra "ferida". As sugestões dos juízes foram semelhantes em todos os aspectos do instrumento, estando as diferenças caracterizadas somente quanto à sinonímia, aos tempos verbais e às preposições, ou seja, diferenças de ordem gramatical.

\section{Retrotradução}

Não houve dificuldades relatadas pelos tradutores nessa fase. Houve pequenas diferenças entre as versões retrotraduzidas e a original, porém sem mudanças de significados ou de conteúdo. Para a finalização da versão adaptada pelas pesquisadoras, apenas a definição do tipo 1 gerou nova discussão com a autora original. Dra. LeBlanc comentou sobre a diferença no seu conteúdo que, na versão adaptada, incluiu explicação mais detalhada sobre a forma de recobertura da LF tipo 1, nos dois casos: lesão linear e retalho cutâneo. Considerando-se que apenas a aproximação de bordas é possível para "cobrir" a lesão no caso de ruptura 
linear e que o reposicionamento do retalho somente é possível quando ele existe, as pesquisadoras consideraram importante detalhar tal explicação cujo conceito parece claro no idioma inglês, mas perde sua veracidade na língua portuguesa. Essa justificativa foi aceita pela autora original e permaneceu na versão adaptada final. Com a aprovação da autora, a versão adaptada do ISTAP Skin Tear Classification foi finalizada como Classificação ISTAP para Lesões por Fricção.

\section{DISCUSSÃO}

Este artigo consiste no relato da primeira etapa de um estudo maior, desenvolvido durante o Programa de Residência em Enfermagem em Cardiopneumologia de Alta Complexidade da EEUSP-InCor, que visou à adaptação cultural e à validação do ISTAP Skin Tear Classification, segundo a metodologia de Beaton et al. ${ }^{9}$, consagrada no meio acadêmico.

Desde a fase de tradução do instrumento, encontraram-se alguns problemas com alguns termos que já trouxeram dificuldades em estudo anterior de adaptação cultural e validação do STAR Skin Tear Classification System 7 , também desenvolvido por uma das pesquisadoras do presente estudo. A expressão skin tear ou diretamente a palavra tear exigiu estudo aprofundado sobre as leis da mecânica para melhor compreensão da etiologia da lesão e estabelecimento da expressão "lesão por fricção" aqui mantida.

Quanto à avaliação das traduções por comitê de juízes, obtiveram-se níveis de IVC bastante baixos (IVC médio: $0,66)$, bem inferior ao valor de corte $(0,9)^{10-13}$, o que poderia ser interpretado como ausência de validade de conteúdo, à crítica imediata. No entanto,esses resultados originaram-se da tradução equivocada do termo flap que aparece nas definições dos três tipos de LF.A versão final foi estabelecida após extensa discussão entre as pesquisadoras, alguns membros do comitê de juízes e a autora original, como indica a literatura em caso de IVC baixo ${ }^{10,11}$. A padronização dos termos "retalho cutâneo" "leito da lesão", respectivamente, para flape wound bed, deveu-se à sua utilização corrente na literatura especializada em dermatologia, cirurgia plástica e estomaterapia. Como trazido por $\mathrm{Neto}^{14}, \mathrm{o}$ retalho cutâneo é composto por um tecido mobilizado ligado ao seu pedículo vascular para manter sua irrigação. A irrigação da pele ocorre por meio de ramos superficiais oriundos da fáscia superficial. Além desses, e conforme descrito na seção Resultados, a definição do tipo 1 - sem perda de pele -, gerou grande discussão, uma vez que é o único tipo de lesão que abrange dois subtipos. Para evitar sua incorreção, as discussões resultaram em detalhamento de seu conteúdo, aprovado por todos, inclusive pela autora original, que pode constatá-lo nas retrotraduções. Assim, considerou-se atestada a validade de conteúdo da versão final em português do ISTAP Skin Tear Classification.

Até o momento, apenas um grupo, na Dinamarca, realizou a tradução e validação do ISTAP Skin Tear Classification System, seguindo a mesma metodologia utilizada no estudo original. A tradução inglês-dinamarquês foi realizada por dois grupos com quatro enfermeiras especialistas em dermatologia, sendo posteriormente analisadas e mescladas para versão final por dois dos autores da adaptação. Em seguida, essa versão foi encaminhada para retrotradução, realizada por dois tradutores, sendo um leigo na área e uma enfermeira com conhecimento do objetivo do estudo. A versão adaptada foi, então, submetida a processo de validação similar ao original. As 30 fotografias originais, pertencentes ao ISTAP, foram aplicadas a 270 profissionais, enfermeiros ( $n=241)$ e médicos (n=29). O nível de concordância apresentado foi moderado para os dois grupos (Kappa de Fleiss $=0.464$ e 0.443, respectivamente), resultado esse que se assemelha àqueles obtidos no estudo origina ${ }^{15}$. Embora não se tenham ainda avaliado as demais propriedades de medida do instrumento em sua versão adaptada ao português do Brasil, as comparações, quer sejam com o estudo original, quer sejam com o único estudo de adaptação cultural e validação publicado, tornam-se difíceis em função dos métodos utilizados em ambos os estudos, distintos daqueles aqui adotados.

\section{Recomendações e limitações do estudo}

O painel ISTAP vem trabalhando com especialistas na área de tratamento de feridas por todos os continentes com o intuito de difundir seu sistema de classificação e demais protocolos de prevenção e tratamento, visando ao alcance de uma linguagem padronizada quanto às LFs, o que facilitaria a valorização do problema e comparação dos resultados obtidos em todo o mundo. Além disso, ele passa por readequação e revisão interna, até mesmo no que diz respeito à nomenclatura das LFs. A adaptação cultural e a validação do sistema de classificação do painel consistem no melhor meio para padronizar a linguagem e difundir o tema por diversos países. Excetuando-se a Dinamarca, primeiro país a adaptar e validar o ISTAP Classification System, apenas Suécia, República Queca, Quebec - Canadá (para o idioma Frânces), Chile e o Brasil encontram-se no processo de adaptação e validação do instrumento. Paralelamente, o 
painel tenta introduzir o termo lesão por fricção ( $\operatorname{lkin}$ tear) na Classificação Estatística Internacional de Doenças e Problemas Relacionados com a Saúde (CID) e no Medical Subject Headings (MeSH).

Dentre as principais limitações do estudo, o tempo reduzido para sua realização influenciou negativamente para que não se apresentasse completo nesse momento. Visto que o trabalho foi realizado como conclusão para o programa de residência, a associação de 60 horas semanais com a pesquisa gera grande sobrecarga de trabalho. A tramitação burocrática para aprovação no comitê de ética em pesquisa e tempo extenso de intervalos de submissão gerou atraso significativo no cronograma proposto. Ademais, a sobrecarga de trabalho dos enfermeiros da instituição, devida à complexidade dos pacientes ali atendidos, retardou ainda mais o processo de coleta de dados, que precisou passar por readequação para ser concluído.

Para disponibilizar um instrumento adaptado, confiável e válido para uso no país, algumas propriedades de medida serão analisadas após a finalização da coleta de dados (confiabilidade inter e intraobservadores e validade de critério concorrente).

\section{CONCLUSÃO}

O ISTAP Skin Tear Classification foi adaptado para a língua portuguesa no Brasil, confirmando-se também sua validade de conteúdo.

\section{CONTRIBUIÇÃO DOS AUTORES}

Conceitualização,Silva CVB; CampaniliTCGF; LeBlanc K; Baranoski S; Santos VLCG.; Metodologia, Silva CVB; LeBlanc K; Santos VLCG; Investigação, Silva CVB; Campanili TCGF; Redação - Primeira versão, Silva CVB; Santos VLCG; Redação - Revisão \& Edição, Silva CVB; LeBlanc K; Santos VLCG.

\section{REFERÊNCIAS}

1. LeBlanc K, Baranoski S. Skin tears: state of the science: consensus statements for the prevention, prediction, assessment, and treatment of skin tears@). Adv Skin Wound Care. 2011;24(9):215. doi: https://doi.org/10.1097/01.ASW.0000405316.99011.95.

2. Carville K, Lewin G, Newall N, Haslehurst $P$, Michael $R$, Santamaria $N$, et al. STAR: a consensus for skin tear classification. Prim Intent Aust J Wound Manag. 2007;15(1):18-27.

3. Amaral AF dos S, Strazzieri-Pulido CS, Santos VLC de G. Prevalence of skin tears among hospitalized patients with cancer. Rev da Esc Enferm. 2012;46(Special):44-50. doi: https://doi.org/10.1590/S0080-62342012000700007.

4. Payne RL, Martin ML. The epidemiology and management of skin tears in older adults. Ostomy Wound Manage. 1990; 26:26-37.

5. Payne RL, Martin ML. Defining and classifying skin tears: need for a common language. Ostomy Wound Manage. 1993;39(5):16-20,22-4,26.

6. Strazzieri-Pulido, KC. Adaptação cultural e validação do instrumento "STAR Skin Tear Classification System" para a língua portuguesa no Brasil [tese]. São Paulo (SP): Universidade de São Paulo, Escola de Enfermagem, Mestrado em Enfermagem na Saúde do Adulto; 2010. doi: https://doi.org/10.11606/D.7.2010.tde-23122010-103305.

7. Strazzieri-Pulido KC, Santos VLC de G, Carville K. Cultural adaptation, content validity and inter-rater reliability of the "STAR Skin Tear Classification System". Rev Lat Am Enfermagem. 2015;23(1):155-61. doi: https://doi.org/10.1590 /2F0104-1169.3523.2537.

8. LeBlanc K, Baranoski S, Holloway S, Langemo D. Validation of a new classification system for skin tears. Adv Ski Wound Care. 2013;26(6):263-5. doi: https://doi.org/10.1097/01. ASW.0000430393.04763.c7.
9. Beaton D, Bombardier C, Guillemin F, Ferraz MB. Recommendations for the cross-cultural adaptation of the DASH \& QuickDASH outcome measures [Internet]. Rosemont: American Academy of Orthopaedic Surgeons and Institute for Work \& Health; 2007 [Acessado 2017 Mar 19]. Disponível em: http://www.dash.iwh.on.ca/sites/dash/ files/downloads/cross_cultural_adaptation_2007.pdf.

10. Alexandre N, Gallasch C. A confiabilidade no desenvolvimento e avaliação de instrumentos de medida na área da saúde. RevEletrônica.2013;15(3):802-9.doi:https://doi.org/10.5216/ ree.v15i3.20776.

11. Polit DF, Beck CT. The content validity index. Are you sure you know what's being reported? Critique and recommendations. Res Nurs Heal. 2006;29(5):489-97. doi: https://doi.org/10.1002/nur.20147.

12. Mcdowell I. Measuring Health: a guide to rating scales and questionnaires. Statistics in medicine. Oxford: Oxford University; 2006. doi: https://doi.org/10.1093/acprof:o so/9780195165678.001.000

13. Souza AC de, Alexandre NMC, Guirardello E de B, Souza AC de, Alexandre NMC, Guirardello E de B. Propriedades psicométricas na avaliação de instrumentos: avaliação da confiabilidade e da validade. Epidemiol e Serviços Saúde. 2017;26(3):649-59. doi: https://doi.org/10.5123/S167949742017000300022

14. Neto NT, Chi A, Ferreira MC. Tratamento cirúrgico das feridas complexas. Rev Med. 2010;89(3/4):147-52.

15. Skiveren J, Bermark S, LeBlanc K, Baranoski S. Danish translation and validation of the International Skin Tear Advisory Panel Skin Tear Classification System. J Wound Care. 2015;24(8):388-92. doi: https://doi.org/10.12968/ jowc.2015.24.8.388. 